\title{
Effective prevention of sorafenib-related vascular damage induced adverse events and maintenance of hepatic function by dried bonito broth and histidine
}

This article was published in the following Dove Press journal: Cancer Management and Research

\author{
Norihiro Sakai ${ }^{1, *}$ \\ Kenya Kamimura ${ }^{1, *}$ \\ Yoko Shinagawa-Kobayashi' \\ Takuro Nagoya' \\ Yusuke Niwa' \\ Masayoshi Ko' \\ Toru Setsu' \\ Akira Sakamaki ${ }^{1}$ \\ Takeshi Yokoo' \\ Satoshi Abe' \\ Hiroteru Kamimura' \\ Soichi Sugitani ${ }^{2}$ \\ Masahiko Yanagi ${ }^{3}$ \\ Shuji Terai' \\ 'Division of Gastroenterology and \\ Hepatology, Graduate School of Medical \\ and Dental Sciences, Niigata University, \\ Niigata, Niigata, Japan; ${ }^{2}$ Department of \\ Gastroenterology, Murakami General \\ Hospital, Murakami, Niigata, Japan; \\ ${ }^{3}$ Department of Gastroenterology, Ojiya \\ General Hospital, Ojiya, Niigata, Japan \\ *These authors contributed equally to \\ this work
}

Correspondence: Kenya Kamimura Division of Gastroenterology and Hepatology, Graduate School of Medical and Dental Sciences, Niigata University,

I-757 Asahimachi-dori, Chuo-ku,

Niigata, Niigata 95।8510, Japan

Tel +8 I25 2272207

Fax +8 I25 2270776

Email kenya-k@med.niigata-u.ac.jp
Background: Sorafenib (SOR) is an anti-angiogenic chemotherapeutic that prolongs the survival rates of patients with hepatocellular carcinoma. However, SOR also damages normal vasculature and causes associated adverse events, including hand-foot syndrome and hypertension (HT). We previously reported in an animal study that vascular damage resulted in the narrowing of the normal vascular dimension area in medaka fish (Oryzias), and histidine (HIS), a major amino acid contained in dried bonito broth (DBB), prevented these changes. Therefore, in the study, we analyzed the effects of DBB and HIS on SORrelated vascular damages and associated adverse events in patients.

Materials and methods: Three-dimensional (3D) vascular images of abdominal regions reconstituted from computed tomography were assessed to compare vascular diameter prior to and following SOR administration in groups receiving SOR monotherapy, $\mathrm{DBB}+\mathrm{SOR}$, and HIS+SOR. The clinical courses of hand-foot syndrome and HT and the toxicities of SOR in biochemical assays were monitored and compared between the groups. Correlations between hepatic function and SOR-related changes in the portal venous area dimension were also assessed.

Results: SOR-related vascular damage revealed narrowing of the normal abdominal vasculature in the human body, which was monitored using 3D images. The damage was ameliorated by DBB and HIS, however, HIS had a more marked effect, particularly on the renal arteries and portal vein (PV). Maintenance of blood flow contributed to the maintenance of total cholesterol, prothrombin time, albumin (ALB), and renal functions. Changes in the 3D vascular area dimension of the PV and level of serum ALB were significantly correlated. The occurrences of the clinical symptoms of hand-foot syndrome and HT were lower in the DBB- and HIS-treated groups.

Conclusion: Our results clearly demonstrate that DBB and HIS prevented SOR-related abdominal vascular damage and effectively maintained hepatic function, and prevented clinical symptoms and toxicity.

Trial registration: This study was registered with the University Hospital Medical Information Network Clinical Trials Registry (UMIN000025937 and UMIN000026898).

Keywords: sorafenib, histidine, dried bonito broth, vascular area dimension, albumin, hepatocellular carcinoma

\section{Plain Language Summary}

Management of the side effects of molecular targeted agents is essential for the patients' quality of life and an improved prognosis. We have reported that sorafenib-induced vascular damage in humans and an in vivo animal model resulted in a decrease in blood flow, which resulted in hand- 
foot syndrome in patients. In addition, we have reported that the ingestion of dried bonito broth maintained the peripheral blood flow in the hands and feet, leading the significant prevention of handfoot syndrome. Following these results, in order to develop an effective method for the prevention of vascular damage due to molecular targeted agents, including sorafenib, we herein report the effect of dried bonito broth and its major content, histidine, on the effective prevention of sorafenib-related abdominal vascular damage induced adverse events and the maintenance of hepatic function.

\section{Introduction}

Multiple kinase inhibitors, including sorafenib (SOR), prolong survival in patients with unresectable advanced hepatocellular carcinoma. ${ }^{1-3}$ However, SOR damages the vasculature, causing hypertension (HT), hand-foot syndrome, and deterioration of hepatic function. Management of the adverse effects of SOR is essential. However, to the best of our knowledge, effective methods to prevent the adverse effects have not been developed to date. We previously reported that reduction of peripheral blood flow is associated with hand-foot syndrome and that dried bonito broth (DBB) can prevent progression of hand-foot syndrome by causing vasodilatation. ${ }^{4}$ In addition, vascular damage to normal vessels by SOR has been confirmed in a medaka fish model. The damage was ameliorated by histidine (HIS), the major amino acid in DBB. ${ }^{5}$ In that study, vascular area dimension was related to SOR-induced vascular damage in the fish. ${ }^{5}$ The dimension of the vasculature in humans can be measured by computed tomography (CT) imaging and is useful in many instances, such as determining the area of small pulmonary vessels and estimating alteration in vascular diameter, which is significantly related to the level of pulmonary arterial pressure in various pulmonary diseases, including chronic obstructive pulmonary diseases. $^{6-9}$ Therefore, in the present study, we examined the relationship between SOR-induced vascular damage in patients treated with SOR and changes in vascular area dimension. In addition, the effects of DBB and HIS on the patients were assessed to determine whether SOR-related vascular damage in humans, HT, hand-foot syndrome, and hepatic dysfunction could be ameliorated by DBB and HIS.

Our study demonstrated that the vascular area of abdominal lesions in SOR-treated patients decreased, leading to HT, hand-foot syndrome, and hepatic dysfunction and that these changes were ameliorated by DBB and HIS.

\section{Materials and methods Study protocol and patients}

The study protocol was approved by the Ethics Committee and Institutional Review Board of Niigata University School of Medicine (clinical trial authorized by Niigata University, number 1482 on August 30, 2012, and number 2016-0082 on March 29, 2017) and was registered with the University Hospital Medical Information Network Clinical Trials Registry (UMIN000025937 and UMIN000026898) on January 31, 2017, and April 20, 2017. Written informed consent was obtained from all patients, and the study was conducted in accordance with the ethical guidance of the 1975 Declaration of Helsinki. Patients with hepatocellular carcinoma scheduled for SOR administration were eligible for the study if they had Eastern Cooperative Oncology performance status of $0-2$ and preserved organ function according to indicators within the liver (Child-Pugh grade A or B). Patients were ineligible if they had dermatological issues or allergies to fish, bonito, or DBB. After informed consent was obtained, the patients were divided into three groups: two study groups of patients starting ingestion of DBB or HIS 1 week prior to SOR initiation (DBB+SOR group and HIS+SOR group) and a control group ingesting no DBB or HIS (SOR group). A three-dimensional (3D) image of the CT scan before SOR administration was used as a control, and images obtained approximately 1 month after SOR administration were assessed (Figure 1).

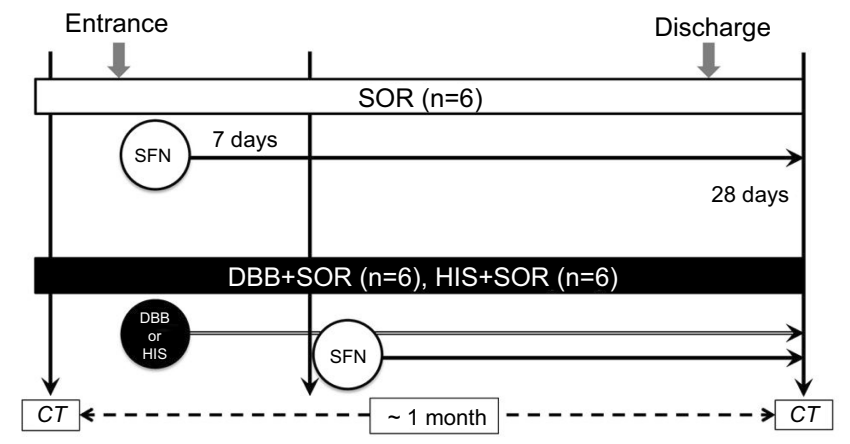

Figure I Study design. The control group $(n=6)$ was treated with SOR without DBB or HIS. The DBB+SOR group $(n=6)$ and the HIS +S OR group $(n=6)$ were treated with SOR with DBB and SOR with HIS.

Abbreviations: SOR, sorafenib; DBB, dried bonito broth; HIS, histidine; CT, computed tomography. 


\section{Dried-bonito broth and histidine}

Bonito (Katsuwonus pelamis) is known as katsuo in Japan, where DBB is easy to obtain and is used to add flavor to food. In this study, the patients in the DBB group ingested $150 \mathrm{~mL}$ of commercially available, highly concentrated rich extract of DBB packs (Honzukuri Ichiban Dashi Kiwami Katsuo) orally, once a day every day, for the entire period of the study, in consistency with the previous study. ${ }^{4}$ The control group consumed normal food, which sometimes (one or two times a week) contained the extract; however, the concentration was approximately 10 -fold lower than that in the pack. The amount of amino acids contained in $150 \mathrm{~mL}$ of $\mathrm{DBB}$ is equal to that in $250 \mathrm{~g}$ of bonito meat. Histidine solution, which was kindly provided by Ajinomoto Co. Inc. (Kawasaki, Kanagawa, Japan), contained $1.65 \mathrm{~g}$ of HIS in $22 \mathrm{~mL}$; DBB contained $0.6 \mathrm{~g}$ of HIS in $150 \mathrm{~mL}$ of solution. The patients in the HIS group ingested $22 \mathrm{~mL}$ of HIS solution orally, once a day every day, for the entire period of the study.

\section{Analysis of vascular area dimension in human}

The vascular area dimension of the vessels was recorded by digital subtraction angiography (DSA) and CT and quantitatively analyzed by using $3 \mathrm{D}$ images of the vessels reconstructed from the $\mathrm{CT}$ images obtained before and 1 month after SOR administration. The images were analyzed by ImageJ software (version 1.6.0_20; National Institutes of Health, Bethesda, MD, USA), and the change in area was expressed as the ratio to the area before initiation of SOR.

\section{Evaluation of adverse events and biochemical analysis}

The occurrence of hand-foot syndrome and changes in blood pressure were monitored by physicians, nurses, or patients every day during the period of SOR administration. NCI-CTCAE (National Cancer Institute Common Terminology Criteria for Adverse Events) version 4.0 was used to assess the grade of hand-foot syndrome. Time-dependent changes in serum biochemical data related to hepatic function were recorded. Serum biochemical factors related to hepatic and renal function were assessed before and 1 month after SOR administration, and the changes were expressed as percentages compared with values before SOR administration.

\section{Statistical analysis}

Categorical variables were analyzed by the MannWhitney-Wilcoxon test. Vascular area dimension and the levels of aspartate aminotransferase (AST), alanine aminotransferase (ALT), total bilirubin (TBIL), total cholesterol (TC), triglycerides (TG), prothrombin time (PT), blood urea nitrogen (BUN), creatinine (CRE), platelets (PLT), and albumin (ALB) in the SOR, DBB+SOR, and HIS +SOR groups were analyzed by Kruskal-Wallis test followed by Dunn's multiple comparison test. Changes in serum ALB and portal vein (PV) area dimension were analyzed by Pearson's correlation test. The data were analyzed with SPSS version 21.0 (Statistical Package for the Social Sciences, IBM Corporation, Armonk, NY, USA). Two-tailed $P$-values $<0.05$ were considered to indicate statistical significance.

Ethics statement

The study protocol was approved by the Ethics Committee and Institutional Review Board of Niigata University School of Medicine (clinical trial authorized by Niigata University, number 1482 on August 30, 2012, and number 2016-0082 on March 29, 2017) and was registered with the University Hospital Medical Information Network Clinical Trials Registry (UMIN000025937 and UMIN000026898) on January 31, 2017, and April 20, 2017. Written informed consent was obtained from all patients, and the study was conducted in accordance with the ethical guidance of the 1975 Declaration of Helsinki.

\section{Results}

\section{Patient characteristics}

Between 2014 and 2018, a total of 18 patients were screened for eligibility, enrolled in this study, and assigned to either the SOR group, DBB+SOR group, or HIS+SOR group. The baseline characteristics of the patients showed no statistical differences between the 3 arms for age, gender, body weight, etiology of the liver disease, presence of liver cirrhosis, Child-Pugh score, stage of HCC, dosage of SOR, period of medication, antitumor response, occurrence of hand-foot syndrome, complication of HT, and period of CT follow up (Table 1).

\section{Impact of SOR on normal blood vessels}

To examine the effect of SOR on normal abdominal blood vessels, vascular area dimension was analyzed in three cases by DSA and 3D images constructed from CT images 
Table I Characteristics of the patients

\begin{tabular}{|c|c|c|c|c|}
\hline \multirow[t]{3}{*}{ Characteristics } & \multicolumn{3}{|l|}{ Group } & \multirow[t]{3}{*}{ MWW test $P$-value } \\
\hline & SOR & SOR+DBB & SOR+HIS & \\
\hline & $n=6$ & $n=6$ & $n=6$ & \\
\hline $\begin{array}{l}\text { Age (years) } \\
\text { Median } \\
\text { Range }\end{array}$ & $\begin{array}{l}72.0 \\
60-80\end{array}$ & $\begin{array}{l}68.0 \\
60-77\end{array}$ & $\begin{array}{l}72.5 \\
48-79\end{array}$ & 0.87 \\
\hline $\begin{array}{l}\text { Gender } \\
\text { Female } \\
\text { Male }\end{array}$ & $\begin{array}{l}1 \\
5\end{array}$ & $\begin{array}{l}1 \\
5\end{array}$ & $\begin{array}{l}0 \\
6\end{array}$ & 0.62 \\
\hline $\begin{array}{l}\text { BW }(\mathrm{kg}) \\
\text { Median } \\
\text { Range }\end{array}$ & $\begin{array}{l}58.7 \\
46.9-76.0\end{array}$ & $\begin{array}{l}61.4 \\
50.1-77.4\end{array}$ & $\begin{array}{l}65.5 \\
48.0-100\end{array}$ & 0.69 \\
\hline $\begin{array}{l}\text { Etiology } \\
\text { HBV infection } \\
\text { HCV infection } \\
\text { Nonalcoholic steatohepatitis } \\
\text { Alcoholic steatohepatitis }\end{array}$ & $\begin{array}{l}0 \\
4 \\
1 \\
1\end{array}$ & $\begin{array}{l}1 \\
2 \\
3 \\
0\end{array}$ & $\begin{array}{l}2 \\
0 \\
4 \\
0\end{array}$ & 1.00 \\
\hline $\begin{array}{l}\text { Cirrhosis } \\
\text { Yes/no }\end{array}$ & $5 / 1$ & $5 / 1$ & $4 / 2$ & 0.79 \\
\hline $\begin{array}{l}\text { Child-Pugh grade } \\
\text { A/B/C }\end{array}$ & $3 / 2 / 0$ & $3 / 2 / 0$ & $0 / 4 / 0$ & 0.85 \\
\hline $\begin{array}{l}\text { Cancer stage } \\
\text { I/II/III/IVA/IVB }\end{array}$ & $0 / 0 / 1 / 3 / 2$ & $0 / 0 / 1 / 2 / 3$ & $0 / 0 / 1 / 4 / I$ & 0.74 \\
\hline $\begin{array}{l}\text { SOR (mg/body) } \\
400 / 600 / 800\end{array}$ & $4 / 2 / 0$ & $3 / 3 / 0$ & $5 / 0 / 1$ & 0.87 \\
\hline $\begin{array}{l}\text { Period of medication (days) } \\
\text { Median } \\
\text { Range }\end{array}$ & $\begin{array}{l}79.0 \\
20-494\end{array}$ & $\begin{array}{l}67.5 \\
21-1040\end{array}$ & $\begin{array}{l}51.5 \\
26-184\end{array}$ & 0.57 \\
\hline $\begin{array}{l}\text { Antitumor response }(n) \\
\text { CR/PR/SD/PD }\end{array}$ & $0 / 1 / 2 / 3$ & $0 / 1 / 3 / 2$ & $0 / 1 / 3 / 2$ & 0.91 \\
\hline HFS (n) & 4 & 0 & I & 0.02 \\
\hline $\begin{array}{l}\text { HFS (grade) } \\
0 / 1 / 2 / 3\end{array}$ & $0 / 2 / 0 / 2$ & 0/0/0/0 & $0 / 0 / 1 / 0$ & \\
\hline HT before SOR & 5 & 5 & 4 & 0.76 \\
\hline HT progression & 4 & 3 & I & 0.11 \\
\hline $\begin{array}{l}\text { Period to CT imaging (days) } \\
\text { Median } \\
\text { Range }\end{array}$ & $\begin{array}{l}33.5 \\
15-40\end{array}$ & $\begin{array}{l}35.5 \\
23-42\end{array}$ & $\begin{array}{l}30.0 \\
28-30\end{array}$ & 0.55 \\
\hline
\end{tabular}

Abbreviations: SOR, sorafenib; HIS, histidine; DBB, dried bonito broth; MWW, Mann-Whitney-Wilcoxon test; BW, body weight; HBV, hepatitis B virus; HCV, hepatitis $C$ virus; $C R$, complete response; PR, partial response; SD, stable disease; PD, progressive disease; HFS, hand-foot syndrome; HT, hypertension; CT, computed tomography. 

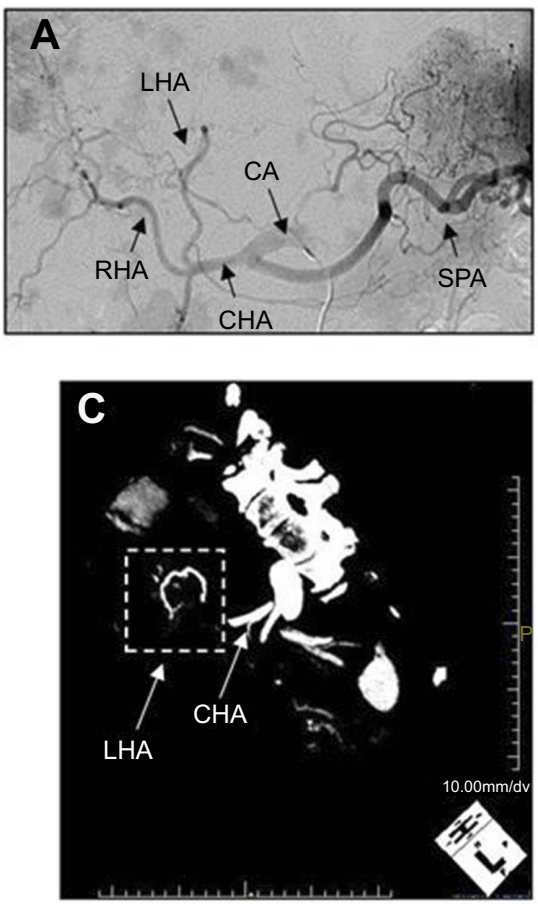
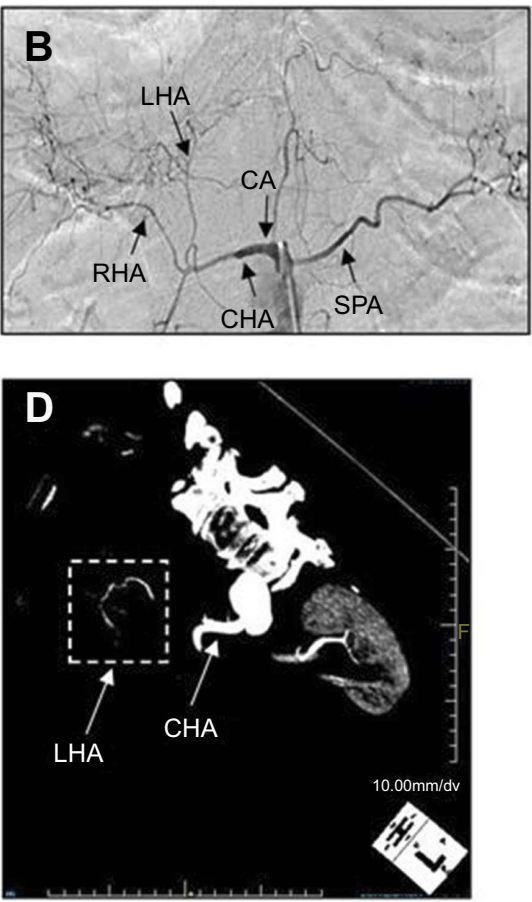

Figure 2 Impact of SOR on normal blood vessels. DSA images of a representative case. (A) before SOR administration and (B) 3 months after SOR administration. 3D images of the vascular structure reconstructed from CT images (C) before SOR administration and (D) 3 months after SOR administration.

Abbreviations: DSA, digital subtraction angiography; SOR, sorafenib; CT, computed tomography; CHA, common hepatic artery; RHA, right hepatic artery; LHA, left hepatic artery; SPA, splenic artery; CA, celiac artery.

before and after SOR administration (Figure 2). Representative images of changes in vascular structure seen by DSA (Figure 2A and B) and 3D images are shown (Figure 2C and D). The major arteries in the abdomen showed significant narrowing in diameter after SOR administration, as shown by DSA, which is consistent with the results obtained from $3 \mathrm{D}$ images. These results indicate that $3 \mathrm{D}$ images from $\mathrm{CT}$ are useful for determining the vascular area dimension of the vessels.

\section{Analyses of SOR-related vascular damages}

To analyze SOR-related vascular damage to the vascular area dimension of various normal vessels in the abdomen, the vascular area dimensions of six patients determined from 3D images taken before and approximately 1 month after SOR administration (Table 1) were assessed and quantified as the ratios to the original areas before the administration of SOR (Figure 3A and B). Changes in the common hepatic artery (CHA), right hepatic artery (RHA), left hepatic artery (LHA), splenic artery (SPA), celiac artery (CA), superior mesenteric artery (SMA), right renal artery (RRA), left renal artery (LRA), and PV were analyzed. Whereas the CA, SMA, and LRA showed no significant changes after SOR administration $(0.97 \pm 0.09,1.01$ \pm 0.08 , and $0.99 \pm 0.03$, respectively), all other vessels examined showed significant decreases in their vascular area dimension compared with the areas before the administration of SOR: CHA (0.89 \pm 0.04$)$, RHA (0.82 $\pm 0.04)$, LHA (0.84 \pm 0.04$)$, SPA $(0.71 \pm 0.07)$, RRA $(0.88$ $\pm 0.07)$, and PV $(0.93 \pm 0.03)(P<0.05)$ (Figure 4). These results show that SOR decreases the vascular area dimension in humans.

\section{Effect of DBB on SOR-related vascular damages}

To examine the effect of DBB on vascular damage in SOR-treated patients, we analyzed the changes in vascular area dimension in SOR-treated patients with DBB administration ( $\mathrm{DBB}+\mathrm{SOR}$ ) (see Materials and Methods). The clinical results for six patients in the $\mathrm{DBB}+\mathrm{SOR}$ group are summarized in Table 1. Representative 3D images of these patients before and approximately 1 month after SOR and DBB administration are shown in Figure $3 \mathrm{C}$ and D. The ratios of the vascular area dimensions from after to before 


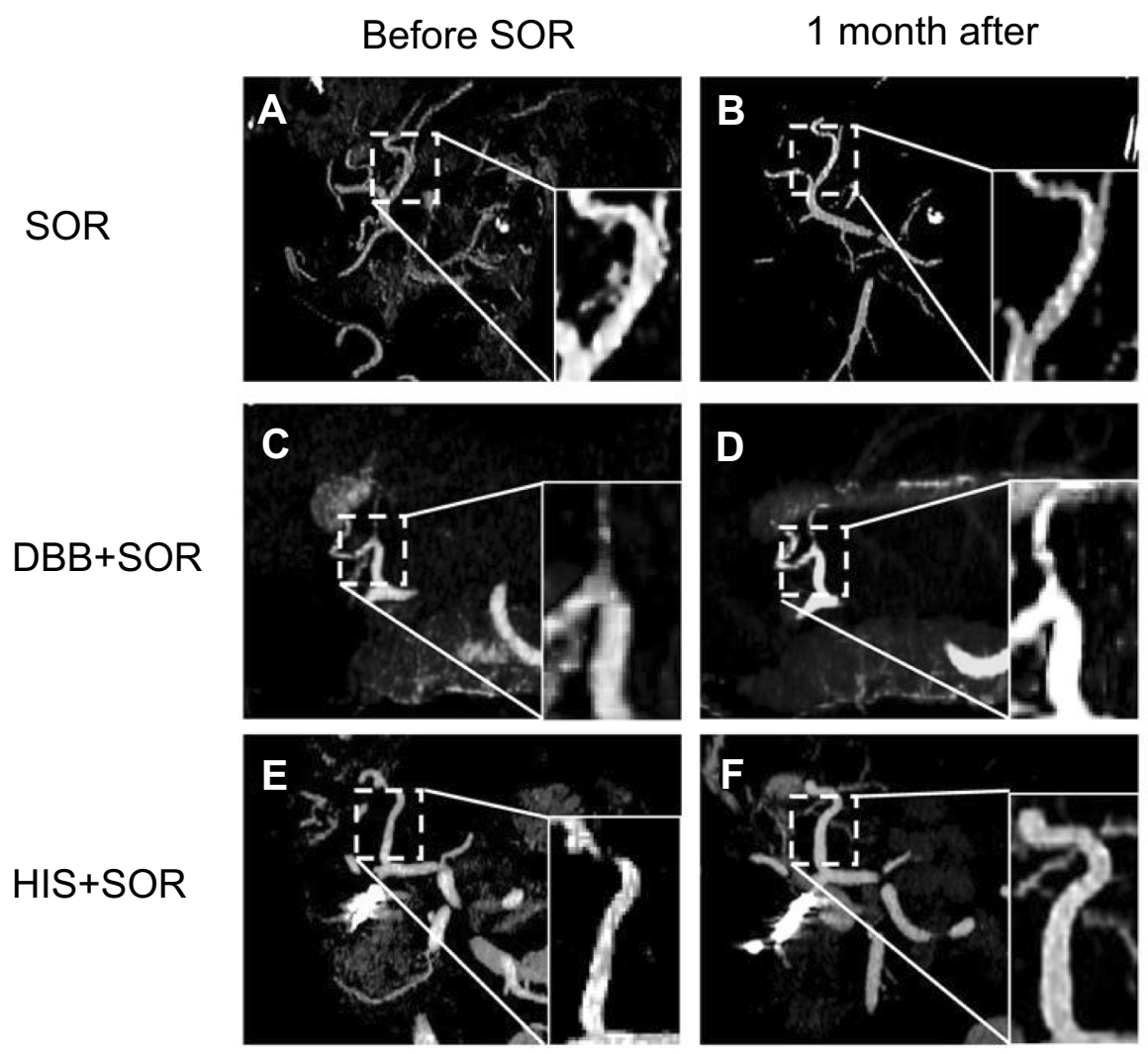

Figure 3 Representative 3D images of vasculature in the SOR, DBB+SOR, and HIS+SOR groups. 3D images of the LHA in the SOR monotherapy group before SOR administration (A) and approximately I month after initiation of SOR (B). DBB+SOR group (C and D). HIS+SOR group (E and F).

Abbreviations: LHA, left hepatic artery; SOR, sorafenib; DBB, dried bonito broth; HIS, histidine.

treatment in these six patients are compared. All vessels assessed showed maintenance or increase in vascular area dimension: CHA (1.27 \pm 0.08$)$, RHA (1.23 \pm 0.10$)$, LHA (1.27 \pm 0.25$)$, SPA (1.26 \pm 0.06$)$, CA (1.05 \pm 0.04$)$, SMA

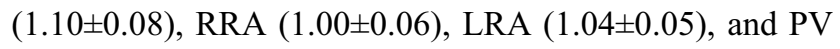
$(0.96 \pm 0.09)$ (Figure 4). Comparison of the values with those in the SOR monotherapy group showed significantly higher changes in CHA $(P<0.05)$, RHA $(P<0.01)$, LHA $(P<0.05)$, and SPA $(P<0.01)$ (Figure 4$)$.

\section{Effect of HIS on SOR-related vascular damages}

To examine the effect of HIS on vascular damage in SOR-treated patients, we analyzed the changes in vascular area dimension in SOR-treated patients with HIS administration (HIS+SOR) (see Materials and Methods). The clinical results for six patients in the SOR+HIS group are summarized in Table 1. Representative 3D images of these patients before and approximately 1 month after SOR and DBB administration are shown in Figure $3 \mathrm{E}$ and $\mathrm{F}$. The ratios of the vascular area dimensions from after to before treatment in these six patients are compared. All vessels assessed showed maintenance or increase in vascular area dimension: CHA (1.40 \pm 0.13$)$, RHA (1.28 \pm 0.11$)$, LHA (1.42 \pm 0.13$)$,

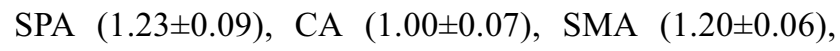
RRA (1.28 \pm 0.12$)$, LRA (1.34 \pm 0.05$)$, and PV (1.27 \pm 0.09 ) (Figure 4). Comparison of the values with those in the SOR monotherapy group showed significantly higher changes in CHA $(P<0.01)$, RHA $(P<0.01)$, LHA $(P<0.05)$, SPA $(P<0.01)$, RRA $(P<0.05)$, LRA $(P<0.01)$, and PV $(P<0.05)$. Maintenance of RRA, LRA, and PV was better in the patients receiving HIS than in those receiving DBB $(P<0.05)$ (Figure 4$)$. The clinical results showed that the occurrence of hand-foot syndrome was lower in the $\mathrm{DBB}+\mathrm{SOR}$ and HIS+SOR groups than in the SOR monotherapy group $(P=0.02)$ and that blood pressure increase was lower in the HIS+SOR group than in the other groups, although this difference was not statistically significant (Table 1).

These results suggest that DBB and HIS contributed to maintain and partly increase the vascular area dimension 


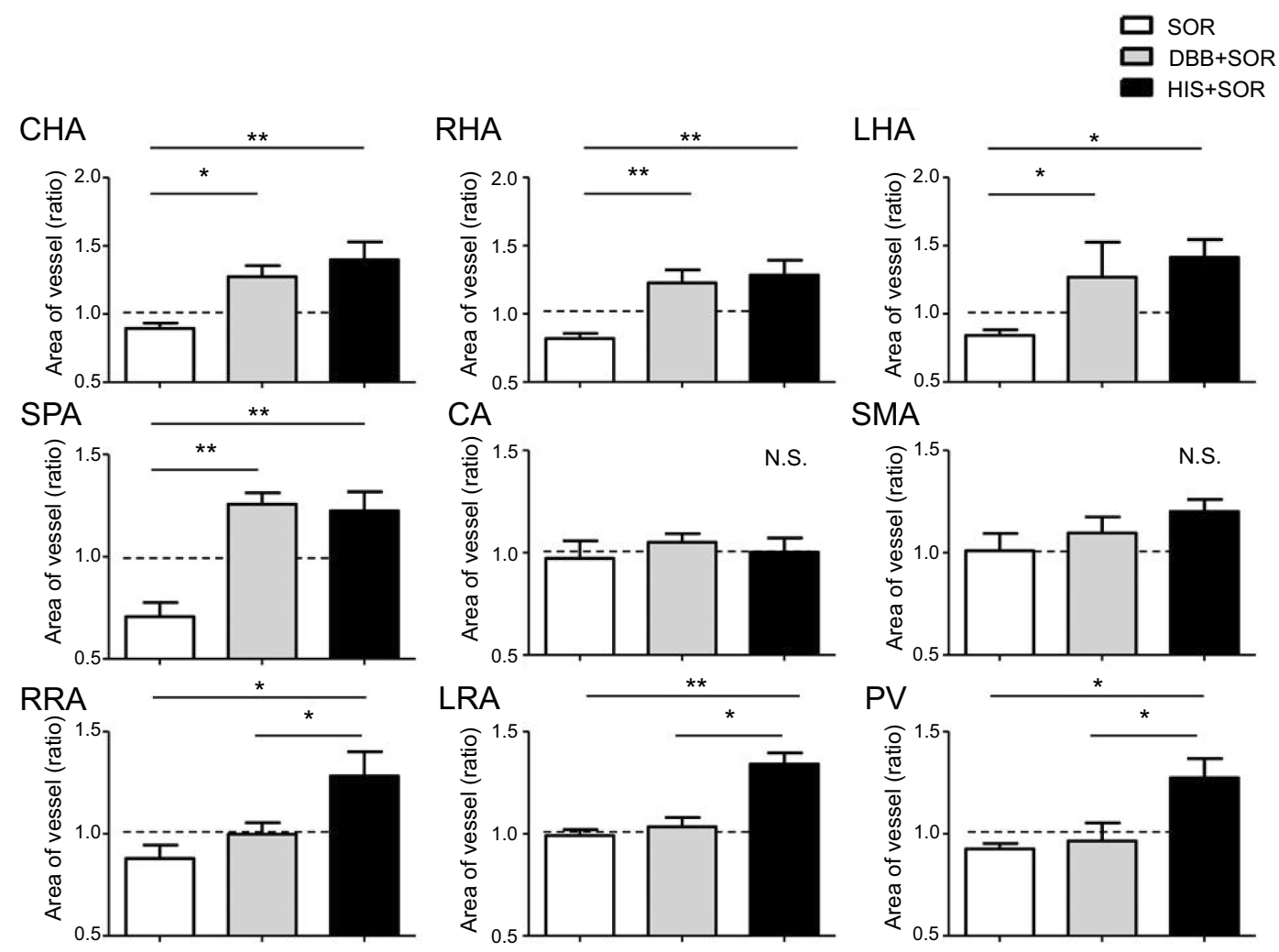

Figure 4 Effect of DBB and HIS on the changes in vascular area dimension caused by SOR. The ratio of vascular area dimension from after to before SOR administration was compared between groups treated with or without DBB or HIS. The values represent means $\pm S D(n=6$ for each group). $* P<0.05$; $* * P<0.01$; NS, not significant. Kruskal-Wallis test followed by Dunn's multiple comparison tests.

Abbreviations: CHA, common hepatic artery; RHA, right hepatic artery; LHA, left hepatic artery; SPA, splenic artery; CA, celiac artery; SMA, superior mesenteric artery; RRA, right renal artery; LRA, left renal artery; PV, portal vein; SOR, sorafenib; DBB, dried bonito broth; HIS, histidine.

of the vessels damaged by SOR administration. This effect may lead to the prevention of adverse events related to the decrease in vascular area dimension by SOR.

\section{Effect of DBB and HIS on SOR-related toxicity}

To examine the effects of DBB and HIS on SOR-related toxicity, biochemical analysis was performed before and 1 month after SOR administration. The baseline levels of the biochemical markers are shown in Table 2. Changes in the values are shown as percentage changes from before to after SOR administration (Figure 5). No significant changes were seen in AST, ALT, TBIL, TG, and PLT in the SOR, DBB+SOR, and HIS+SOR groups. TC increased in the DBB $(7.07 \pm 13.25 \%)$ and HIS (11.45 $\pm 8.54 \%)$ groups, even after SOR administration, and decreased in the SOR monotherapy group $(-12.76 \pm 8.13 \%)$; however, these changes were not statistically significant. PT (\%) decreased in the SOR monotherapy group (-14.85 $\pm 6.82 \%)$ and increased in the DBB+SOR and HIS+SOR groups by $2.57 \pm 3.10 \%$ and $1.71 \pm 5.64 \%$, respectively. BUN increased by $11.5 \pm 8.86 \%$ in the SOR monotherapy group and decreased in the $\mathrm{DBB}+\mathrm{SOR}$ and $\mathrm{HIS}+\mathrm{SOR}$ groups by $-2.11 \pm 7.54 \%$ and $-18.5 \pm 7.48 \%$, respectively. CRE increased by $6.83 \pm 8.1 \%$ in the SOR monotherapy group and showed a milder increase in the $\mathrm{DBB}+\mathrm{SOR}$ group $(0.95 \pm 4.27 \%)$ and a decrease in the HIS+SOR group ( $-14.6 \pm 2.56 \%)$. These results suggest that treatment with DBB and HIS resulted in better maintenance of TC and PT and renal function than did SOR monotherapy.

\section{Effect of maintenance of PV blood flow on the level of serum ALB}

To determine whether management of adverse events and vascular damage could contribute to the maintenance of nutrition status and hepatic function, we examined changes in the serum level of ALB in the SOR, DBB+SOR, and HIS + SOR groups 1 month after administration of SOR (Figure 6A). SOR monotherapy resulted in a significant decrease $(-10.3 \pm 2.32 \%)$ in the serum level of ALB. The 
Table 2 Background of serum biochemical analysis

\begin{tabular}{|c|c|c|c|c|}
\hline \multirow[t]{3}{*}{ Serum markers } & \multicolumn{3}{|l|}{ Group } & \multirow[t]{3}{*}{ MWW test $P$-value } \\
\hline & SOR & SOR+DBB & SOR+HIS & \\
\hline & $n=6$ & $n=6$ & $n=6$ & \\
\hline AST (IU/L) & & & & 0.25 \\
\hline Median & 60 & 45.5 & 52.5 & \\
\hline Range & $30-233$ & $26-157$ & $23-114$ & \\
\hline ALT (IU/L) & & & & 0.67 \\
\hline Median & 43 & 34 & 52 & \\
\hline Range & $11-125$ & $17-137$ & $18-118$ & \\
\hline TBIL (mg/dL) & & & & 0.14 \\
\hline Median & I & 0.75 & 0.8 & \\
\hline Range & $0.6-1.6$ & $0.4-1.4$ & $0.4-1.6$ & \\
\hline TC (mg/dL) & & & & 0.23 \\
\hline Median & 188.5 & 163.5 & $|3|$ & \\
\hline Range & $122-244$ & $128-183$ & $114-188$ & \\
\hline TG (mg/dL) & & & & 0.95 \\
\hline Median & 51.5 & 66 & 72 & \\
\hline Range & $34-159$ & $57-126$ & $68-86$ & \\
\hline PT (\%) & & & & 0.49 \\
\hline Median & 84 & 79.5 & 90 & \\
\hline Range & $70-100$ & $68-104$ & $6 I-128$ & \\
\hline BUN (mg/dL) & & & & 0.10 \\
\hline Median & 16 & 14.5 & 17.5 & \\
\hline Range & $14-27$ & $10-20$ & $9-25$ & \\
\hline CRE (mg/dL) & & & & 0.89 \\
\hline Median & 0.79 & 0.74 & 0.8 & \\
\hline Range & $0.57-0.9$ & $0.5 \mathrm{I}-\mathrm{I} .28$ & $0.6-0.98$ & \\
\hline PLT $\left(\times 10^{4} / \mu \mathrm{L}\right)$ & & & & 0.91 \\
\hline Median & 13.7 & 13.5 & 11.6 & \\
\hline Range & $11.4-32.9$ & $3.8-80.4$ & $10.5-30.5$ & \\
\hline
\end{tabular}

Abbreviations: SOR, sorafenib; HIS, histidine; DBB, dried bonito broth; MWW, Mann-Whitney-Wilcoxon test; AST, aspartate aminotransferase; ALT, alanine aminotransferase; TBIL, total bilirubin; TC, total cholesterol; TG, triglycerides; PT, prothrombin time; BUN, blood urea nitrogen; CRE, creatinine; PLT, platelets.

DBB + SOR group had a milder reduction in serum ALB $(-1.91 \pm 2.15 \% ; P<0.05)$, and the HIS+SOR group had an increase in serum ALB $(2.55 \pm 3.11 \% ; P<0.05)$. As the PV area dimension was effectively maintained in the DBB + SOR and HIS+SOR groups (Figure 4), analysis of the correlation between changes in serum ALB and PV area dimension was performed (Figure 6B). The results suggested a significant correlation between changes in serum ALB and PV area dimension, indicating that the effect of DBB and HIS on maintenance of PV flow contributed to the hepatic function reserve in patients receiving SOR treatment.

\section{Discussion}

SOR is known to contribute to prolonged survival rate in patients with unresectable advanced hepatocellular carcinoma by inhibiting multiple kinases associated with tumor growth, including vascular endothelial growth factor, vascular endothelial growth factor receptor, and PLT-derived growth factor receptor. ${ }^{1-3}$ SOR causes adverse events associated with 

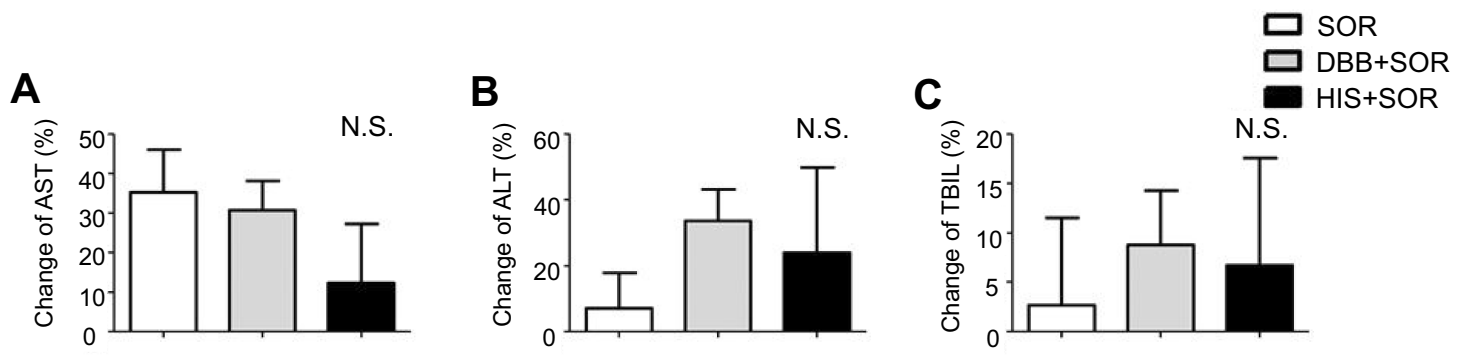

D

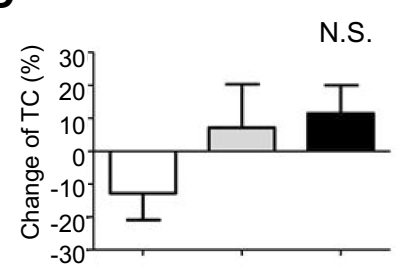

G

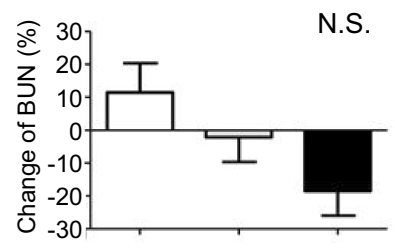

E

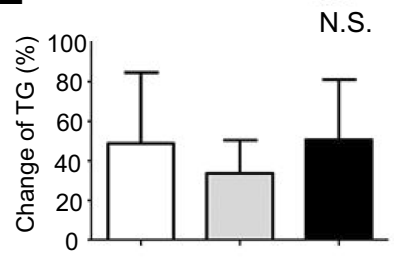

H

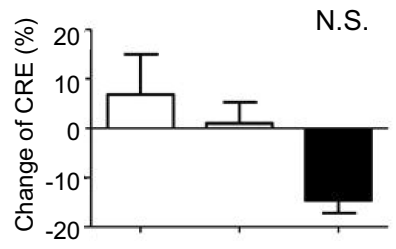

F

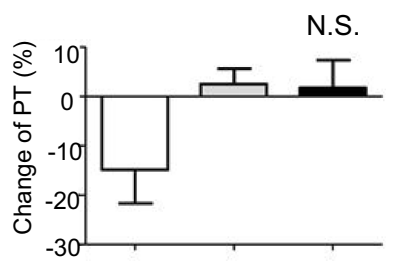

I

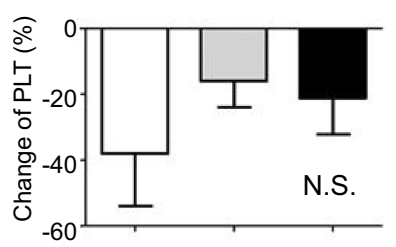

Figure 5 Effect of DBB and HIS on the changes in serum biochemical factors caused by SOR.

Notes: (A-I) Changes in serum biochemical factors from before to after SOR administration were compared between the groups treated with or without DBB or HIS. The values represent means \pm SD ( $n=6$ for each group). N.S., not significant, Kruskal-Wallis test followed by Dunn's multiple comparison tests.

Abbreviations: AST, aspartate aminotransferase; ALT, alanine aminotransferase; TBIL, total bilirubin; TC, total cholesterol; TG, triglycerides; PT, prothrombin time; BUN, blood urea nitrogen; CRE, creatinine; PLT, platelets; SOR, sorafenib; DBB, dried bonito broth; HIS, histidine
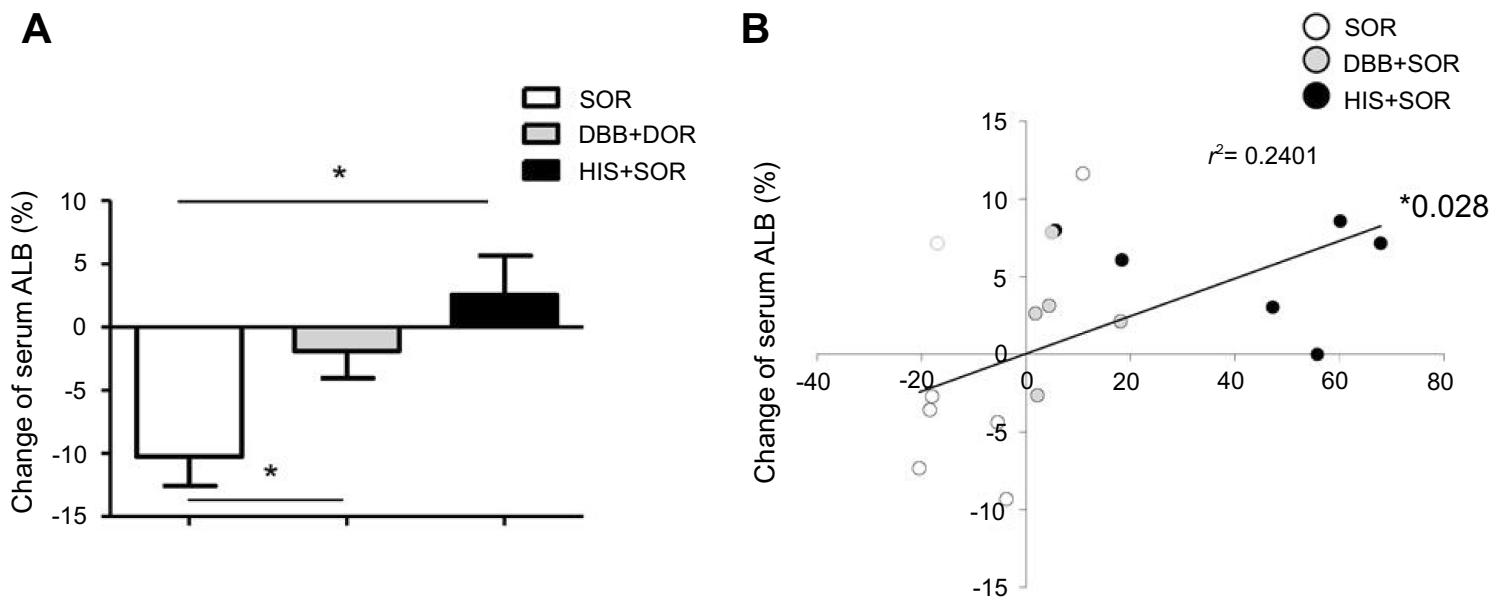

Change of PV area dimension (\%)

Figure 6 Effect of DBB and HIS on serum ALB level and PV area dimension.

Notes: (A) Changes in serum ALB from the levels before SOR administration were compared between the groups treated with or without DBB or HIS. The values represent means \pm SD ( $n=6$ for each group). $* P<0.05$. Kruskal-Wallis test followed by Dunn's multiple comparison test. (B) Changes in serum ALB and PV area dimension. The bold black line shows the trend line, and analysis of the correlation between changes in serum ALB and PV area dimension was performed. $* P<0.05$, Pearson's correlation test. Abbreviations: ALB, albumin; PV, portal vein; SOR, sorafenib; DBB, dried bonito broth; HIS, histidine. 
vascular damage, including HT, hand-foot syndrome, and deterioration of hepatic function. Management of these adverse events is essential. We previously reported that SORrelated hand-foot syndrome was associated with a decrease in peripheral blood flow and that DBB improved the maintenance of blood flow, leading to the alleviation of hand-foot syndrome. ${ }^{10}$ Vascular damage from SOR has also been reproduced in the vascular area dimension of GFP-labeled vessels in the transgenic medaka model. ${ }^{5}$ HIS, the major component among the amino acids contained in the bonito extract, ${ }^{11}$ alleviated this vascular damage in the medaka fin by maintaining the vascular area dimension without growing new vessels and exhibited no tumor cell growth. ${ }^{5}$ The results from an animal model and in vitro study have suggested that DBB and HIS may contribute to the maintenance of blood flow with no evidence of tumor growth. Therefore, in the present study, we examined the effects of DBB and HIS on SOR-related vascular damage by analyzing the vascular area dimension of patients treated with SOR.

Our study demonstrated that the vascular area in SOR-treated patients decreased in various vessels, which was consistent with the results of the previous in vivo animal study (Figure 2), and that this change was ameliorated by DBB and HIS (Figures 3 and 4). The maintenance of abdominal arterial flow supported the digestive and renal arteries, decreasing the frequency of blood pressure increase caused by the mechanisms of the renal regulation of arterial blood pressure. ${ }^{12,13}$ This phenomenon also contributed to maintaining renal function following the administration of SOR, as indicated by the levels of BUN and CRE (Figure 5). These changes in the major vessels also decreased the occurrence of handfoot syndrome by supporting arterial flow in the peripheral vessels.

The mechanism underlying the recovery of the vascular area dimension by DBB and HIS may be through the $\mathrm{H} 1$ and $\mathrm{H} 2$ histamine receptors and attenuation of sympathetic output via the central $\mathrm{H} 3$ receptor. ${ }^{14,15}$ Nozawa et al reported that DBB increased peripheral blood flow in humans ${ }^{16,17}$ and cerebral blood flow in mice ${ }^{11}$ and rats. ${ }^{18}$ Treatment of spontaneously hypertensive rats with HIS decreased HT by attenuating sympathetic output via the central H3 receptor. ${ }^{19}$ The mechanisms by which HIS affects vascular dilatation may include the following: 1) HIS accumulates in mast cells, which synthesize histamine to directly stimulate the $\mathrm{H} 1$ receptor in vascular structures and causing dilatation of the vasculature; 2) HIS is a precursor of carnosine, which has been shown to scavenge reactive oxygen species, thus contributing to the dilatation of the vessels ${ }^{20}$; and 3) orally administered L-histidine is converted into histamine as an effector in the central nervous system and has hypotensive effects via $\mathrm{H} 3$ receptors as histamine itself is unable to cross the bloodbrain barrier. The oral administration of L-histidine had no effect on peripheral histamine concentration, whereas histamine contained in the cerebrospinal fluid increased. ${ }^{19}$ These results suggest that HIS in DBB may cause vasodilatation exerted via central nerve signaling.

The present study also showed that DBB and HIS maintained the PV vascular area, with HIS having a more marked effect (Figure 4). This effect of HIS on venous dilatation has been reported as a histamine-mediated vasodilatation ${ }^{14,21}$ and has been used clinically to maintain endothelium and smooth muscle function in the human saphenous vein transplant. ${ }^{15}$ In addition, as previously reported, ${ }^{22-24}$ our study showed that the maintenance of PV flow contributed to the management of hepatic function, including serum levels of ALB, during chemotherapy (Figure 6).

These results suggest that DBB and HIS maintain blood flow in various vessels, including the renal arteries and PV, by vasodilatation, leading to the improved management of hepatic function, renal function, and blood pressure. Although a larger clinical study will be necessary to determine the effects of DBB and HIS on various kinase inhibitors, the results of the present study suggest that DBB and HIS can ameliorate the adverse events of SORrelated vascular damage in humans, leading to improved outcomes in patients treated with SOR. In addition, successful management in the HIS group, which tended to comprise cases with poorer hepatic reserve function, indicates that the combination of HIS with SOR may effectively prevent and manage clinical symptoms from toxicities induced by SOR. Regarding the effect of DBB and HIS on the antitumor effect of SOR, we have shown that DBB is effective for maintaining peripheral blood flow and preventing HFS induced by SOR treatment; in addition, no differences in tumor response were observed between the DBB group and the control group. ${ }^{4}$ This result is consistent with the results obtained with DBB and HIS in this study (Table 1). We also assessed these phenomena in the basic animal research and showed that HIS only assisted in preventing SOR-related vascular damage in normal vessels and did not have positive effects on new developing abnormal vessels in an animal model, ${ }^{5}$ with no increase of cell proliferation evident; therefore, it can be used without the loss of the antitumor effect of SOR. 
A limitation of the present study is that the sample size was modest, as there was a small number of patients in each group and whom serum biochemical and CT data for three-dimensional images at appropriate time points were available. In addition, as this was the first pilot study, we designed this clinical study to administer DBB and/or HIS for the duration of the first cycle of SOR administration. Therefore, challenges and future directions of this study involve the assessment of the efficacy of DBB and HIS in larger groups in multiple centers and of the long-term administration of DBB and/or HIS. In addition, as shown in our previous study, ${ }^{4}$ blood flow in the vessels of the hand and foot can be examined by Doppler ultrasound, and the combination of CT and ultrasound may further contribute to the monitoring of adverse events and develop an effective prevention methodology using DBB and HIS.

\section{Conclusion}

The results of this study clearly demonstrated that DBB and HIS prevented SOR-related abdominal vascular damage and effectively maintained hepatic function and prevented clinical symptoms and toxicities. Further clinical assessment in larger groups and including patients treated with other molecular targeted medicines will encourage us to develop safe and effective methods to prevent the adverse effects of these medicines and to maintain hepatic function.

\section{Data sharing statement}

The authors do not intend to share the individual deidentified participant data and the data and document will be available on the University Hospital Medical Information Network Clinical Trials Registry website (UMIN000025937 and UMIN000026898).

\section{Acknowledgments}

The authors would like to thank Takao Tsuchida of the Division of Gastroenterology and Hepatology at the Niigata University for his excellent assistance in preparing the figures. They also thank Enago for the critical reading of the manuscript and English language editing. The authors would like to thank for the financial support from the Mishima Kaiun Memorial Foundation which, in part, supported the laboratory research.

\section{Author contributions}

All authors contributed toward data analysis, drafting and revising the paper, gave final approval of the version to be published and agree to be accountable for all aspects of the work.

\section{Disclosure}

The authors report no conflicts of interest in this work.

\section{References}

1. Llovet JM, Ricci S, Mazzaferro V, et al. Sorafenib in advanced hepatocellular carcinoma. N Engl J Med. 2008;359:378-390. doi:10.1056/ NEJMoa0708857

2. Cheng AL, Kang YK, Chen Z, et al. Efficacy and safety of sorafenib in patients in the Asia-Pacific region with advanced hepatocellular carcinoma: a phase III randomised, double-blind, placebo-controlled trial. Lancet Oncol. 2009;10:25-34. doi:10.1016/S1470-2045(08)70285-7

3. Furuse J, Ishii H, Nakachi K, Suzuki E, Shimizu S, Nakajima K. Phase I study of sorafenib in Japanese patients with hepatocellular carcinoma. Cancer Sci. 2008;99:159-165. doi:10.1111/j.1349-7006.2007.00648.x

4. Kamimura K, Shinagawa-Kobayashi Y, Goto R, et al. Effective prevention of sorafenib-induced hand-foot syndrome by dried-bonito broth. Cancer Manag Res. 2018;10:805-813. doi:10.2147/CMAR.S159370

5. Shinagawa-Kobayashi Y, Kamimura K, Goto R, et al. Effect of histidine on sorafenib-induced vascular damage: analysis using novel medaka fish model. Biochem Biophys Res Commun. 2018;496:556-561. doi:10.1016/ j.bbrc.2018.01.057

6. Uejima I, Matsuoka S, Yamashiro T, Yagihashi K, Kurihara Y, Nakajima Y. Quantitative computed tomographic measurement of a cross-sectional area of a small pulmonary vessel in nonsmokers without airflow limitation. Jpn J Radiol. 2011;29:251-255. doi:10.1007/s11604010-0551-9

7. Matsuoka S, Washko GR, Yamashiro T, et al. Pulmonary hypertension and computed tomography measurement of small pulmonary vessels in severe emphysema. Am J Respir Crit Care Med. 2010;181:218-225. doi:10.1164/rccm.200908-11890C

8. Matsuoka S, Washko GR, Dransfield MT, et al. Quantitative CT measurement of cross-sectional area of small pulmonary vessel in COPD: correlations with emphysema and airflow limitation. Acad Radiol. 2010;17:93-99. doi:10.1016/j.acra.2009.07.022

9. Usuba A, Yamashiro T, Handa $H$, et al. Quantitative computed tomography measurement of tracheal cross-sectional areas in relapsing polychondritis: correlations with spirometric values. Respiration. 2015;90:468-473. doi:10.1159/000441303

10. Kamimura K, Shinagawa Y, Ogawa K, et al. Effective prevention of hand-foot syndrome by the consumption of dried bonito broth. Gan To Kagaku Ryoho. 2016;43:463-465.

11. Honda M, Nozawa Y, Ishizaki T, Kuroda M. Ingestion of bonito extract ameliorates peripheral blood flow in mice loaded from over crowding stress. Biomed Res. 2009;30:129-135.

12. Kimura G, Saito F, Kojima S, et al. Renal function curve in patients with secondary forms of hypertension. Hypertension. 1987;10:11-15.

13. Digne-Malcolm H, Frise MC, Dorrington KL. How do antihypertensive drugs work? Insights from studies of the renal regulation of arterial blood pressure. Front Physiol. 2016;7:320. doi:10.3389/fphys.2016.00320

14. Romero SA, McCord JL, Ely MR, et al. Mast cell degranulation and de novo histamine formation contribute to sustained postexercise vasodilation in humans. $J$ Appl Physiol. 2017;122:603-610. doi:10.1152/japplphysiol.00633.2016 
15. Bas M, Luther B, Knopf A, Suvorava T, Kojda G. Preservation of endothelial and smooth muscle function of human saphenous vein transplants. Exp Clin Transplant. 2016;14:86-92.

16. Macedo LT, Lima JP, Dos Santos LV, Sasse AD. Prevention strategies for chemotherapy-induced hand-foot syndrome: a systematic review and meta-analysis of prospective randomised trials. Support Care Cancer. 2014;22:1585-1593. doi:10.1007/s00520-014-2129-z

17. Nozawa Y, Ishizaki T, Kuroda M, Noguchi T. Effect of dried-bonito broth intake on peripheral blood flow, mood, and oxidative stress marker in humans. Physiol Behav. 2008;93:267-273. doi:10.1016/j. physbeh.2007.08.021

18. Honda M, Yamada H, Nozawa Y, Ishizaki T, Kuroda M, Noguchi T. Consumption of bonito extract suppresses the decrease in cerebral blood flow in stroke-prone spontaneously hypertensive rats. Biomed Res. 2010;31:251-258.

19. Toba H, Nakamori A, Tanaka Y, et al. Oral L-histidine exerts antihypertensive effects via central histamine $\mathrm{H} 3$ receptors and decreases nitric oxide content in the rostral ventrolateral medulla in spontaneously hypertensive rats. Clin Exp Pharmacol Physiol. 2010;37:62-68. doi:10.1111/j.1440-1681.2009.05227.x
20. Nagai K, Tanida M, Niijima A, et al. Role of L-carnosine in the control of blood glucose, blood pressure, thermogenesis, and lipolysis by autonomic nerves in rats: involvement of the circadian clock and histamine. Amino Acids. 2012;43:97-109. doi:10.1007/s00726-012-1251-9

21. Romero SA, Ely MR, Sieck DC, et al. Effect of antioxidants on histamine receptor activation and sustained postexercise vasodilatation in humans. Exp Physiol. 2015;100:435-449. doi:10.1113/EP085030

22. Okuda K, Ohnishi K, Kimura K, et al. Incidence of portal vein thrombosis in liver cirrhosis. An angiographic study in 708 patients. Gastroenterology. 1985;89:279-286.

23. Giannitrapani L, Grana W, Licata A, Schiavone C, Montalto G, Soresi M. Nontumorous portal vein thrombosis in liver cirrhosis: possible role of beta-blockers. Med Princ Pract. 2018;27:466-471. doi: $10.1159 / 000492893$

24. Choi SH, Kwon JH, Kim KW, et al. Measurement of liver volumes by portal vein flow by Doppler ultrasound in living donor liver transplantation. Clin Transplant. 2017;31:e13050. doi:10.1111/ ctr. 13050

\section{Publish your work in this journal}

Cancer Management and Research is an international, peer-reviewed open access journal focusing on cancer research and the optimal use of preventative and integrated treatment interventions to achieve improved outcomes, enhanced survival and quality of life for the cancer patient.
The manuscript management system is completely online and includes a very quick and fair peer-review system, which is all easy to use. Visit http://www.dovepress.com/testimonials.php to read real quotes from published authors. 